\title{
Análise da Estatística Nosológica Nacional 2009-2013. Grupo Português de Dermatologia Pediátrica
}

\author{
Eduarda Osório Ferreira \\ Assistente Hospitalar Graduada de Dermatologia e Venereologia/Graduated Consultant, Dermatology and Venereology; \\ Serviço de Dermatologia, Centro Hospitalar de Vila Nova de Gaia/Espinho, Portugal \\ Grupo Português de Dermatologia Pediátrica/Pediatric Dermatology Portuguese Group
}

RESUMO - O Grupo Português de Dermatologia Pediátrica elaborou uma análise da estatística nosológica nacional no período de 2009 a 2013, baseada nos dados compilados de 12 centros a nível nacional. Foram estudados dados referentes a 22.354 primeiras consultas. Os eczemas foram o grupo de doenças mais frequentemente observado -5.104 pacientes $(22,3 \%)$, seguido dos nevos - $4.678(20,4 \%)$, infecções - $4.651(20,3 \%)$, doenças das faneras - $2.770(12,1 \%)$, doenças vasculares - $1.066(4,7 \%)$, e doenças da pigmentação - $1.033(4,5 \%)$. Foi ainda feita uma análise comparativa entre a estatística actual e aquela realizada pelo Grupo há 10 anos. Este tipo de estudos parece-nos importante porque permite conhecer a realidade nacional relativamente à prevalência das doenças cutâneas da infância, eventuais variações ao longo dos anos e estabelecer comparações com estudos realizados noutros países. Finalmente, poderão ajudar a melhorar a medicina preventiva.

PALAVRAS-CHAVE - Criança; Doenças da Pele/estatística e dados numéricos; Portugal.

\section{National Pediatric Dermatology Nosologic Statistics Analysis 2009-2013. Pediatric Dermatology Portuguese Group}

ABSTRACT - The Pediatric Dermatology Portuguese Group performed a survey of the national nosologic statistic of the years 2009 2013, collecting data from 12 national hospitals with a consultation of pediatric dermatology. A total of 22,354 patients were studied. Eczemas were the most common diseases, with 5, 104 patients (22.3\%), followed by nevi - 4,678 (20.4\%), infections - 4,651 (20.3\%), adnexal diseases - 2,770 (12.1\%), vascular diseases - 1,066 (4.7\%) and pigmentary disorders - 1,033 (4.5\%). Furthermore, a comparative analysis was performed with nosologic statistics from ten years ago. In our opinion these studies are important since they allow us to know the national prevalence of pediatric cutaneous diseases, their evolution and aid us in establishing comparison with studies from other countries. Finally, they may help to improve preventive medicine.

KEY-WORDS - Child; Portugal; Skin Diseases/statistics and numerical data.

\section{INTRODUÇÃO}

O Grupo Português de Dermatologia Pediátrica (GPDP) propôs-se elaborar uma análise retrospectiva da estatística nosológica nacional no período de 2009 a 2013, baseada nos dados compilados de 12 centros a nível nacional onde existia, à data, uma consulta organizada da subespecialidade de Dermatologia Pediátrica: Hospital de Braga, Hospital Curry Cabral (Centro Hospitalar Lisboa Central), Hospital
Santa Maria (Centro Hospitalar Lisboa Norte), Centro Médico-Cirúrgico de Lisboa, Centro Hospitalar e Universitário de Coimbra, Hospital Geral de Santo António (Centro Hospitalar do Porto), Centro Hospitalar de S. João, Hospital Egas Moniz, (Centro Hospitalar Lisboa Ocidental), Hospital de Faro (Centro Hospitalar do Algarve), Hospital Distrital da Figueira da Foz, Hospital Distrital de Santarém, Centro Hospitalar de Vila Nova de Gaia /Espinho.
Correspondência: Eduarda Osório Ferreira Grupo Português de Dermatologia Pediátrica

Serviço de Dermatologia - Centro Hospitalar de Vila Nova de Gaia/Espinho

Rua Conceição Fernandes - 4434 -502 Vila Nova de Gaia, Portugal

E-mail: eduardaosorioferreira@gmail.com
Recebido/Received

28 Fevereiro/28 February 2016

Aceite/Accepted

1 Maio/1 May 2016 


\section{Dermatologia Pediátrica}

Foi ainda feita uma análise comparativa entre a estatística actual e aquela realizada pelo Grupo há 10 anos, relativa ao período de 1998-2003, apresentada pela Dra. Maria João Rodrigo em nome do GPDP, no Congresso Nacional da SPDV, em Novembro de 2003.

\section{MATERIAL E MÉTODOS}

O grupo dispõe de uma base de dados em formato Excel onde é feito o registo de todas as patologias que motivam uma primeira consulta, divididas por grandes grupos nosológicos.

$\mathrm{Na}$ análise foram incluídos todos os pacientes com idade inferior a 16 anos referenciados à consulta de Dermatologia Pediátrica desde 1 de Janeiro de 2009 a 31 de Dezembro de 2013.

O diagnóstico foi estabelecido com base em critérios estandardizados de diagnóstico clínico e, quando necessário, apoiado por estudos laboratoriais, testes cutâneos e/ou exames histopatológicos.

Os grandes grupos nosológicos analisados neste estudo foram: eczemas, nevos, infecções cutâneas, doenças das faneras e vasculares, alterações da pigmentação, genodermatoses, prurigos e doenças eritemato-papulo-descamativas.

\section{RESULTADOS}

Neste período de cinco anos foi possível reunir os dados referentes a 22.354 primeiras consultas, correspondendo a 22.878 patologias.

Os eczemas foram o grupo de doenças mais frequentemente observado - 5.104 pacientes $(22,3 \%)$, seguido dos nevos - $4.678(20,4 \%)$, infecções - $4.651(20,3 \%)$, doenças das faneras - $2.770(12,1 \%)$, doenças vasculares - 1.066 $(4,7 \%)$ e doenças da pigmentação - 1.033 (4,5\%). Comparativamente, as genodermatoses - 591 pacientes $(2,6 \%)$, os prurigos - $524(2,3 \%)$ e as doenças eritematodescamativas - $496(2,2 \%)$ tiveram uma incidência reduzida. Outros grupos nosológicos (correspondentes a 1.212 pacientes) - mastocitoses, urticárias, eritemas, reacções adversas a fármacos, doenças da hipoderme, doenças causadas por agentes físicos, doenças bolhosas, doenças das mucosas, doenças das células de Langerhans/macrófagos, anomalias do desenvolvimento, doenças metabólicas/endócrinas e reumatológicas/multissistémicas -, quando considerados individualmente, tiveram uma frequência igual ou inferior a $0,5 \%$.

À data da consulta, 753 pacientes $(3,3 \%)$ não apresentavam qualquer doença cutânea. (Fig. 1)

Analisando os grupos nosológicos individualmente, verificamos que no dos eczemas o subgrupo mais frequente foi o do eczema atópico, com 3.214 pacientes (63\%), seguido do eczema seborreico com 658 (12,9\%). Outras formas de doença neste grupo incluíram a pitiríase alba - 336 doentes $(6,6 \%)$, o eczema de contacto - $306(6 \%)$, o eczema desidrótico - $116(2,3 \%)$, o eczema numular - $95(1,9 \%)$, a dermatose plantar juvenil - $94(1,8 \%)$ e a dermite perioral

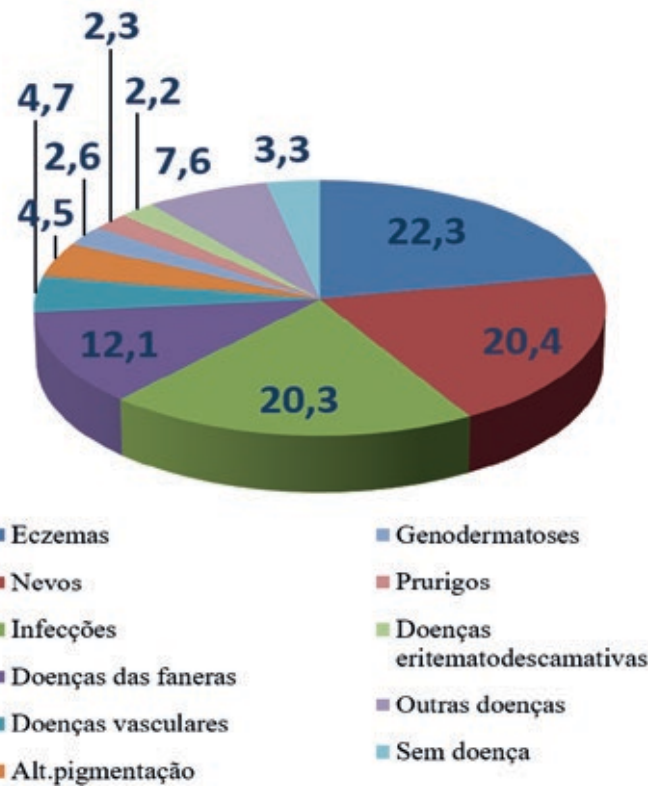

Figura 1 - Distribuição percentual dos grandes grupos nosológicos.

- $47(0,9 \%)$. Em 238 pacientes (4,6\%) observaram-se outras manifestações de eczema/dermite.

Quanto aos nevos predominaram os melanocíticos $4.324(92,4 \%)$, quando comparados com os epidérmicos - $354(7,6 \%)$. Os nevos melanocíticos adquiridos - 2158 $(49,9 \%)$, foram observados num $\mathrm{n}^{\circ}$ superior ao dos nevos congénitos - $1.884(43,6 \%)$, o mesmo se passando com os nevos epidérmicos verrucosos - $186(52,5 \%)$, quando comparados com os nevos sebáceos - $154(43,5 \%)$. Outros tipos de nevos melanocíticos foram observados em 282 pacientes $(6,5 \%)$ e outros tipos de nevos epidérmicos em $14(4 \%)$.

No grupo das doenças infecciosas predominaram largamente aquelas provocadas por vírus - 3.067 (65,9\%), seguidas, por ordem decrescente, as infecções por fungos $-1.154(24,8 \%)$, parasitas - $250(5,4 \%)$ e bactérias - 179 $(3,8 \%)$. Outros agentes foram diagnosticados em $0,1 \%$.

Das viroses observadas, 1.600 doentes $(52,2 \%)$ tinham moluscos contagiosos, $1.394(45,5 \%)$ verrugas e $37(1,2 \%)$ infecções do grupo herpes. Outras viroses foram diagnosticadas em 36 pacientes $(1,1 \%)$.

Das infecções fúngicas, em 901 pacientes $(78,1 \%)$ foram diagnosticadas tinhas. Destas, 595 (66\%) eram tinhas do couro cabeludo, $84(9,3 \%)$ da pele glabra, $82(9,1 \%)$ dos pés, $77(8,5 \%)$ das unhas, $29(3,2 \%)$ da face, $14(1,6 \%)$ eram tinhas incógnitas e, em 10 doentes cada $(2,2 \%)$, tinha crural e das mãos.

Outras formas de infecção por fungos - 253 doentes $(21,9 \%)$ incluíram a pitiríase versicolor em 165 pacientes (14,3\% das infecções fúngicas totais), as candidíases em $77(6,7 \%)$, a onicomicose não-dermatofítica em $8(0,7 \%)$, outras em $3(0,3 \%)$.

A parasitose dominante foi a escabiose - em 212 pacientes $(84,8 \%)$, seguida da pediculose em $29(11,6 \%)$; 


\section{Dermatologia Pediátrica}

outras parasitoses foram diagnosticadas em $9(3,6 \%)$.

Finalmente, as piodermites foram identificadas em 165 doentes $(92,2 \%)$ das infecções provocadas por bactérias e as corinebacterioses em $10(5,6 \%)$; outras infecções bacterianas foram diagnosticadas em 4 (2,2\%).

As doenças da glândula sebácea foram aquelas mais frequentemente observadas no grupo da patologia das faneras -1755 pacientes $(63,4 \%)$, seguidas das doenças das unhas - 466 (16,8\%), do cabelo/pêlo - 430 (15,5\%) e da glândula sudorípara - 119 (4,3\%).

A acne foi diagnosticada em 1730 doentes $(98,6 \%$ das doenças da glândula sebácea); 25 (1,4\%) manifestaram outras doenças.

A hiperidrose foi o diagnóstico em 81 doentes $(68,1 \%$ das doenças da glândula sudorípara), a hidradenite supurativa em $21(17,6 \%)$ e a miliária em $12(10,1 \%) ; 5$ doentes $(4,2 \%)$ tiveram outras formas de doença.

No subgrupo nosológico das doenças do cabelo/pêlo, as alopécias diagnosticadas em 321 pacientes $(74,7 \%)$ foram as mais frequentemente observadas, nomeadmante alopécia areata 278 - 86,6\%, alopécia androgenética 16 - 0,5\%, alopécia de tracção 14 - 0,4\%, alopécia cicatricial $13-0,4 \%$. Seguiram-se os deflúvios - 41 (9,5\%), a hipertricose/hirsutismo - 40 (9,3\%), as alterações da haste capilar $11(2,6 \%)$ e outras manifestações ocorreram em 17 (3,9\%).

As patologias das unhas incluíram, por ordem decrescente de frequência, as doenças congénitas (mau-alinhamento, paquioniquia) - em 206 pacientes (44,2\%), a onicocriptose - em 100 (21,5\%), a traquioniquia - em 16 $(3,4 \%)$ e a onicólise - em 9 (1,9\%); onicotilomania, leuconiquia, melanoniquia, pontuado ungueal, paraqueratose pustulosa, entre outras, constituíram 29\% (135 doentes) das doenças deste subgrupo.

A lesão mais frequentemente observada no grupo das doenças vasculares foi o hemangioma comum da infância - 544 pacientes (52\%), seguido das malformações (mancha vinho-do-porto, mancha salmão, aranha vascular, telangiectasia, cutis marmorata, outras) - 437 pacientes (41\%); outras doenças vasculares (granuloma piogénico, nevo anémico, angioqueratoma, malformação linfática) foram observadas em 85 (7\%).

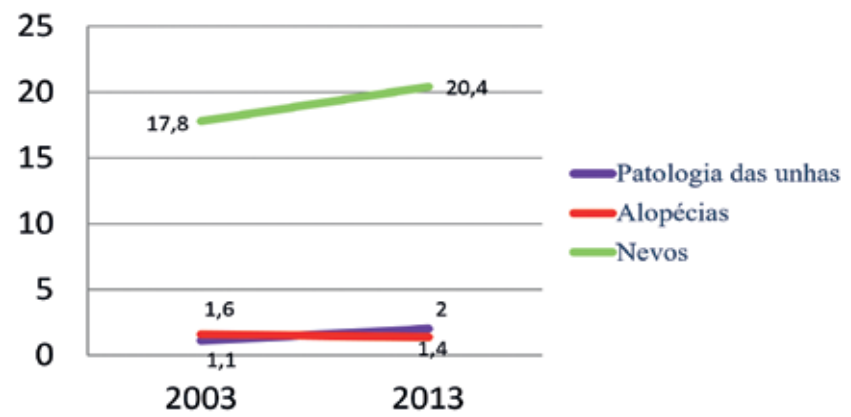

Figura 2 - Análise comparativa das estatísticas de 2003 e 2013 (valores percentuais): patologia das unhas, alopécias e nevos.

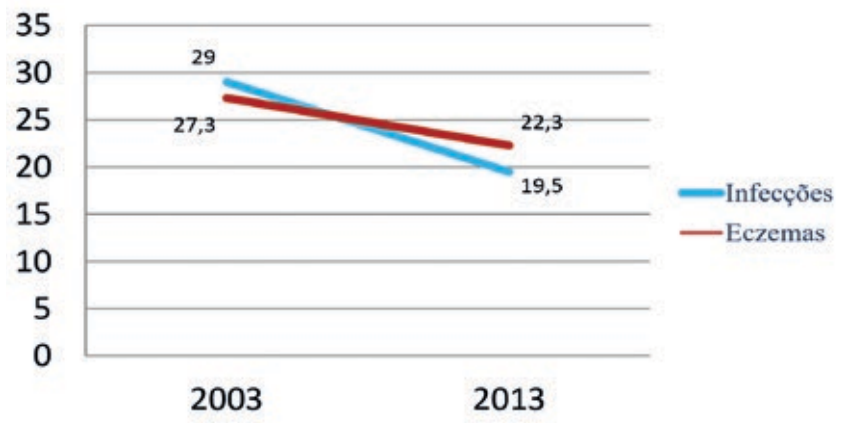

Figura 3 - Análise comparativa das estatísticas de 2003 e 2013 (valores percentuais): infecções e eczemas.

Nas alterações da pigmentação predominou o subgrupo das hipopigmentações - 636 pacientes $(61,6 \%)$. As hiperpigmentações representaram 38,4\% (397 pacientes).

$O$ vitiligo foi a doença mais prevalente no primeiro subgrupo - 337 doentes (53\%), seguido da hipopigmentação pós-inflamatória - 101 (15,8\%), do nevo acrómico - 99 $(15,6 \%)$ e da hipomelanose de lto - 10 (1,6\%); outras doenças foram diagnosticadas em 89 pacientes (14\%).

No segundo subgrupo das alterações da pigmentação prevaleceram, por ordem decrescente de frequência, a mancha café-com-leite - 153 doentes (38,5\%), as hiperpigmentações pós-inflamatórias - 95 (23,9\%), o nevo de Becker - 45 (11,3\%); outras doenças (fitofotodermatite, efélides, lentigos, lentiginose, incontinência pigmentar, entre outras) foram diagnosticadas em 104 (26,3\%).

As genodermatoses mais observadas foram a queratose pilar - 314 doentes $(53,1 \%)$, as facomatoses - $101(17,1 \%)$ (neurofibromatoses 82-81\%, esclerose tuberosa 19-19\%), as ictioses - $86(14,6 \%)$ e a epidermólise bolhosa - 57 $(9,6 \%)$; outras genodermatoses foram observadas em 33 $(5,6 \%)$.

De 524 pacientes $(2,3 \%)$ com formas de prurigo, 512 doentes $(97,7 \%)$ tinham prurigo estrófulo, 10 (1,9\%) prurigo nodular e $2(0,4 \%)$ outras formas da doença.

No grupo das doenças eritemato-papulo-descamativas a psoríase foi a patologia mais observada - 334 pacientes $(67,3 \%)$, seguida do líquen estriado - $48(9,7 \%)$, pitiríase rósea - $43(8,7 \%)$, líquen nítido - 28 (5,6\%), pitiríase liquenóide - $20(4,1 \%)$, líquen plano - 17 (3,4\%), pitiríase rubra pilar - 6 (1,2\%).

Comparando a estatística actual com aquela realizada pelo Grupo há 10 anos, verificamos que a patologia das unhas e as alopécias se mantiveram em percentagens relativamente estáveis e os nevos sofreram um aumento moderado (Fig. 2), enquanto que nos eczemas e nas infecções se verificou uma descida ligeira a moderada (Fig. 3). De salientar que no grupo das infecções a redução se deveu, sobretudo, às infecções por fungos. Por outro lado, as doenças vasculares, a acne e as alterações da pigmentação sofreram um aumento significativo, duplicando os seus números percentuais (Fig. 4). 


\section{Dermatologia Pediátrica}

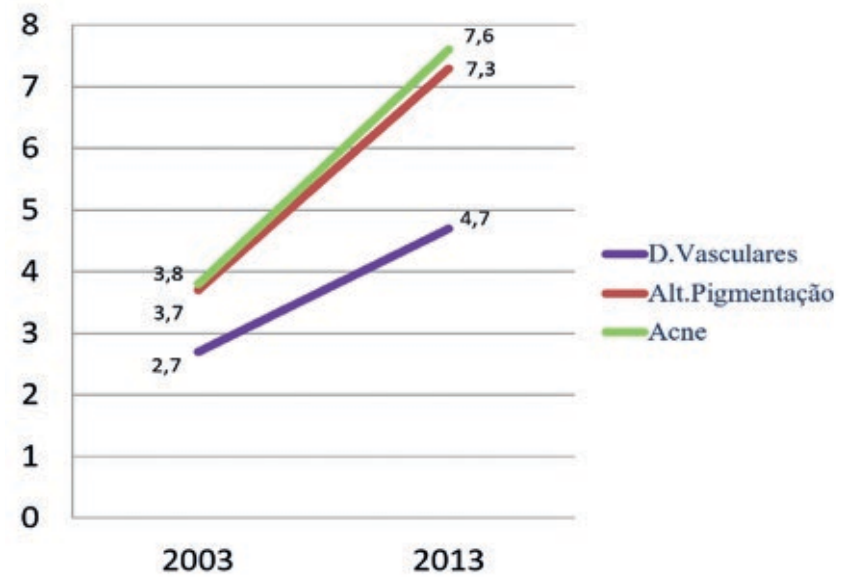

Figura 4 - Análise comparativa das estatísticas de 2003 e 2013 (valores percentuais): doenças vasculares, alterações da pigmentação, acne.

\section{DISCUSSÃO}

Na nossa análise, e à semelhança de outros estudos publicados, encontramos uma elevada prevalência de eczemas/dermatites, ${ }^{1-5}$ nevos $^{6,7}$ e infecções. ${ }^{7-15}$ Noutros estudos, contudo, a dermatose mais prevalente foi a acne ${ }^{16,17}$ ou, ainda, as dermatoses alérgicas. ${ }^{18}$

Por outro lado, dermatoses frequentes como a dermite das fraldas, os exantemas víricos e as parasitoses como a escabiose e a pediculose, entre outras, foram observadas numa frequência significativamente reduzida, provavelmente por estas crianças serem tratadas na sua maioria pela pediatria e medicina geral e familiar.

Comparativamente à estatística realizada pelo Grupo há 10 anos, a actual revela-nos que os nevos sofreram um aumento moderado como motivo de referenciação a uma consulta de Dermatologia Pediátrica. Este facto poderá ser explicado, por um lado, por um receio crescente de cancro cutâneo que advém de um maior alerta da comunidade médica e civil e, por outro - e paradoxalmente -, pela excessiva exposição solar, fruto de uma banalização de férias balneares e da valorização social atribuída a um "aspecto bronzeado". A acne, as doenças vasculares e as alterações da pigmentação sofreram um aumento significativo, duplicando os seus números percentuais. Outros estudos corroboram os nossos achados. ${ }^{19-21}$

De salientar que na literatura estão disponíveis muito poucos estudos epidemiológicos das doenças dermatológicas na faixa pediátrica, particularmente realizados na Europa.

Este tipo de estudos parece-nos importante porque permite conhecer e compreender a realidade nacional relativamente à prevalência das doenças cutâneas da infância, eventuais variações na sua incidência ao longo dos anos e estabelecer comparações com estudos realizados noutros países. Finalmente, e em particular no que diz respeito a doenças potencialmente controláveis, poderão desempenhar um papel importante no estabelecimento de estratégias e políticas de medicina preventiva e saúde pública, representando por isso um papel-chave para o desenvolvimento de um programa de cuidados de saúde infantil eficaz.

Conflitos de interesse: Os autores declaram não possuir conflitos de interesse. Suporte financeiro: $O$ presente trabalho não foi suportado por nenhum subsídio ou bolsa. Confidencialidade dos dados: Os autores declaram ter seguido os protocolos do seu centro de trabalho acerca da publicação dos dados de doentes.

Conflicts of interest: The authors have no conflicts of interest to declare. Financing Support: This work has not received any contribution, grant or scholarship. Confidentiality of data: The authors declare that they have followed the protocols of their work center on the publication of data from patients.

\section{REFERÊNCIAS}

1. Nanda A, Al-Hasawi F, Alsaleh QA. A prospective survey of Pediatric Dermatology clinic patients in Kuwait: analysis of 10.000 cases. Pediatr Dermatol. 1999; 16:6-11.

2. Shibeshi D. Pattern of skin diseases at the Ethio- Swedish pediatric hospital, Addis Abeba, Ethiopia. Pediatr Dermatol. 2000; 17:357-9.

3. Wenk C, Itin PH. Epidemiology of pediatric dermatology and allergology in the region of Argau, Switzerland. Pediatr Dermatol. 2003; 20:482-7.

4. Shrestha R, Shrestha D, Dhakal AK, Shakya A, Shah SC, Shakya $\mathrm{H}$. Spectrum of pediatric dermatoses in tertiary care center in Nepal. Nepal Med Coll J. 2012; 14:146-8.

5. Wisuthsarewong W, Viravan S. Analysis of skin diseases in a referral pediatric dermatology clinic in Thailand. J Med Assoc Thai. 2000 ;83:999-1004.

6. Casanova JM, Sanmartin V, Soria X, Baradad M, Martí RM, Font A. Childhood dermatosis in a dermatology clinic of e general university hospital in Spain. Actas Dermosifiliogr. 2008; 99:111-8.

7. El-Khateeb EA, Lotfi RA, Abd Elaziz KM, El-Shiekh SE. Prevalence of skin diseases among primary schoolchildren in Damietta, Egypt. Int J Dermatol 2014; 53:609-16.

8. Gül Ü, Çakmak SK, Gonül M, Kiliç A, Bilgili S. Pediatric skin disorders encountered in a Dermatology outpatient clinic in Turkey. Pediat. Dermatol. 2008;25:277-8.

9. Leite I, Moreira A, Guedes R, Baptista A, Osório Ferreira E. Pediatric skin disorders in a Portuguese hospital. Eur J Pediatr Dermatol. 2010; 20:191-4.

10. Kacar SD, Ozuguz P, Polat S, Manav V, Bukulmez A, Karaca S. Epidemiology of pediatric skin diseases in the mid-western Anatolian region of Turkey. Arch Argent Pediatr. 2014; 112:421-7.

11. Jawade SA, Chugh VS, Gohil SK, Mistry AS, Umrigar DD. A clinico-etiological study of dermatoses in pediatric age group in tertiary health care center in South Gujarat Region. Indian J Dermatol. 2015; 60:635.

12. Kiprono SK, Muchunu JW, Masenga JE. Skin diseases in 


\section{Dermatologia Pediátrica}

pediatric patients attending a tertiary dermatology hospital in Northern Tanzania: a cross-sectional study. BMC Dermatol. 2015 10; 15:16.

13. Sacchidanand S, Sahana MS, Asha GS, Shilpa K. Pattern of pediatric dermatoses at a referral centre. Indian J Pediatr. 2014; 81:375-80.

14. El-Khateeb EA. The spectrum of paediatric dermatoses in a university hospital in Cairo, Egypt. J Eur Acad Dermatol Venereol. 2011 ; 25:666-72.

15. Al-Mendalawi MD. Ibrahim JG. Pattern of dermatoses in Iraqi children. East Mediterr Health J. 2012; 18:365-71.

16. Tamer E, Ilhan MN, Polat M, Lenk N, Alli N. Prevalence of skin diseases among pediatric patients in Turkey. J Dermatol. $2008 ; 35: 413-8$.
17. Sanfilippo AM, Barrio V, Kulp-Shorten C, Callen JP. Common pediatric and adolescent skin conditions. J Pediatr Adolesc Gynecol. 2003; 16:269-83.

18. Ferreira FR, Nascimento LF, Cirvidiu DC. Prevalence of pediatric dermatoses in a university hospital in southeastern Brazil. An Bras Dermatol. 201 1;86:477-82.

19. Bhate K, Williams HC. Epidemiology of acne vulgaris. $\mathrm{Br}$ J Dermatol. 2013;168:474-85.

20. Sidoroff A. Epidemiologie kutaner vaskulärer neu- und fehlbildungen im kindesalter. Handchir Mikrochir Plast Chir. 2009; $41: 65-9$.

21. Jablonski NG, Chaplin G. Human skin pigmentation, migration and disease susceptibility. Philos Trans R Soc Lond B Biol Sci. 2012; 367:785-92. 